\title{
Association between ultrasound measurements of muscle thickness, pennation angle, echogenicity and skeletal muscle strength in the elderly
}

\author{
Eva Maria Strasser • Thomas Draskovits • \\ Markus Praschak • Michael Quittan • \\ Alexandra Graf
}

Received: 2 August 2012 / Accepted: 10 February 2013 / Published online: 2 March 2013

(C) American Aging Association 2013

\begin{abstract}
The increase of elderly in our society requires simple tools for quantification of sarcopenia in inpatient and outpatient settings. The aim of this study was to compare parameters determined with musculoskeletal ultrasound (M-US) with muscle strength in young and elderly patients. In this prospective, randomised and observer blind study, 26 young (24.2 \pm 3.7 years) and 26 old (age $67.8 \pm 4.8$ years) patients were included. Muscle thickness, pennation angle and echogenicity of all muscles of musculus quadriceps were measured by M-US and correlated with isometric maximum voluntary contraction force (MVC) of musculus quadriceps. Reproducibility of MUS measurements as well as simple and multiple regression models were calculated. Of all measured M-US variables the highest reproducibility was found for
\end{abstract}

Clinical trial Registration Number: NCT00933192

E. M. Strasser · T. Draskovits • M. Quittan

Institute of Physical Medicine and Rehabilitation, Social

Medical Centre South, Kaiser Franz Joseph Hospital,

Kundratstrasse 3, 1100 Vienna, Austria

E. M. Strasser $(\bowtie) \cdot$ T. Draskovits $\cdot$ M. Praschak •

M. Quittan

Karl Landsteiner Institute of Remobilisation and Functional Health, Social Medical Centre South, Kaiser Franz Joseph

Hospital, Kundratstrasse 3, 1100 Vienna, Austria

e-mail: eva-maria.strasser@wienkav.at

A. Graf

Institute of Medical Statistics, Medical University of

Vienna, Währinger Gürtel 18-20, Vienna 1090, Austria measurements of thickness (intraclass correlation coefficients, 85-97\%). Simple regression analysis showed a highly significant correlation of thickness measurements of all muscles of musculus quadriceps with MVC in the elderly and in the young. Multiple regression analysis revealed that thickness of musculus vastus medialis had the best correlation with MVC in the elderly. This study showed that measurement of muscle thickness, especially of musculus vastus medialis, by M-US is a reliable, bedside method for monitoring the extent of sarcopenia.

Keywords Musculoskeletal ultrasound · Muscle thickness $\cdot$ Pennation angle $\cdot$ Echogenicity $\cdot$ Skeletal muscle strength $\cdot$ Sarcopenia

$\begin{array}{ll}\text { Abbreviations } \\ \text { M-US } & \begin{array}{l}\text { Musculoskeletal ultrasound } \\ \text { MVC }\end{array} \\ & \begin{array}{l}\text { Maximum isometric voluntary contraction } \\ \text { force }\end{array} \\ \text { ICC } & \text { Intra-class correlation coefficient } \\ \text { CSA } & \text { Cross-sectional area } \\ \text { BMI } & \text { Body mass index }\end{array}$

\section{Introduction}

Sarcopenia is characterised by significantly reduced skeletal muscle mass, muscle strength and functional physical performance (Cruz-Jentoft et al. 2010). Studies 
report that until the age of 80 healthy people lose about $30-40 \%$ of their skeletal muscle mass and acute or chronic illness could even increase this dramatic muscle loss (Frontera et al. 2000). It has been shown that these changes in skeletal muscle mass are closely related to a progressive loss of skeletal muscle strength in ageing people (Hughes et al. 2001). Moreover, low muscle strength is predictive for immobilisation, physical disability and increased mortality (Rantanen et al. 1999; Cooper et al. 2010; Taekema et al. 2010). However, active muscle strength testing in elderly patients is often limited by several factors as for example immobilisation of the patient, pain, injury, surgical interventions, cognitive impairments or technical complexity. Therefore, there is growing interest in development of objective parameters correlating to skeletal muscle strength to early detect skeletal muscle loss and monitor therapeutic interventions.

Skeletal muscle strength is highly depending on muscle mass, composition and architecture. The cross-sectional area (CSA) of a muscle, a parameter for muscle mass, is related to its muscle strength (Ikai and Fukunaga 1968; Maughan et al. 1983b; Young et al. 1985; Fukunaga et al. 2001). Muscle architecture, described by pennation angle, gives information about muscle strength as the larger the pennation angle, the more contractile material can be packed within a certain volume and thereby increases the muscle's capacity to produce force (Kawakami et al. 1993). Manini et al. report that muscle disuse result in altered composition, characterised by a significant increased accumulation of intramuscular fat which is accompanied by a significant loss of muscle strength (Manini et al. 2007). Nevertheless, it has to be mentioned that neural factors, as motor unit recruitment, firing frequency, motor unit synchronisation or inter-muscular coordination also have a high impact on muscle strength.

Until now, magnetic resonance tomography or computer tomography are the gold standards to measure CSA and muscle composition. Although these techniques provide precise results, they are expensive, cause radiation exposure and are of restricted availability. Musculoskeletal ultrasound (MUS) is another bedside imaging method used to assess muscle mass as well as architecture and composition. The major drawback of this method is the limited image size which prevents the direct measurement of CSA of large muscles. However, muscle thickness, defined as the distance between two fascias, is easily determined with M-US. It has been shown that muscle thickness is highly and significantly correlated to the CSA of a muscle (Abe et al. 1997). Moreover, it has been shown that muscle loss of intensive care unit patients could be monitored by thickness measurements of musculus rectus femoris and musculus intermedius (Gruther et al. 2008). Pennation angles of musculus vastus lateralis and musculus intermedius were significantly correlated to the CSA (Rutherford and Jones 1992). Information about the muscle composition can be gathered by quantification of muscle echogenicity. Pillen et al. have found a significant correlation between M-US echogenicity and interstitial fibrous tissue achieved from muscle biopsies of dogs (Pillen et al. 2009). Moreover, it has been shown that atrophying processes correlate with increased accumulation of intramuscular fat and fibrous tissue resulting in higher ultrasound echogenicity (Reimers et al. 1993).

There are several publications using M-US to measure thickness, pennation angle or echogenicity. However, completeness of data concerning reproducibility of all measurable M-US parameters in all muscles of musculus quadriceps is missing. Therefore, we intended to study the reproducibility of M-US measurements of thickness, pennation angle and echogenicity in all muscles of musculus quadriceps in young patients with healthy muscles as well as in old patients with sarcopenic muscles. Secondly, it was of interest to measure maximum isometric voluntary contraction (MVC) force of musculus quadriceps to correlate it with M-US parameters. Moreover, regression analysis was used to evaluate which M-US parameter showed the best correlation to MVC. This study reveals that especially muscle thickness is highly correlated to muscle strength in the young and the old group.

\section{Material and methods}

\section{Study design}

This prospective, randomised, and observer blind study was conducted in conformity with the Declaration of Helsinki and the protocol was approved by the local ethical committee. Patients were recruited from the outpatient clinic of the Institute of Physical Medicine and Rehabilitation of the 
Social Medical Centre South-Kaiser Franz Joseph Hospital and gave their written informed consent for participation. Patients were eligible for inclusion if they were either between 18 and 35 (young group) or between 60 and 80 (elderly group) years of age. Exclusion criteria were neuromuscular diseases, prosthesis or fractures of the lower extremities, injuries of joints or ligaments of the lower extremities, acute or chronic pain of the lower extremities, cognitive impairments, malign diseases or pregnancy.

Assignment

One leg of the patient was randomly assigned for investigation and examinations were applied on two separate days, two times by two observers in random order. The randomization was based on a randomization list provided by the Institute of Medical Statistics of the Medical University of Vienna.

Blinding procedures

The observer conducting the randomization and data analysis was not further involved in the measurement of M-US parameters or muscle strength.

Assessment of anthropometric data, physical performance and handgrip strength

Baseline variables including age, weight, height, physical activity and handgrip strength were assessed. Body weight and height were measured during the first examination and expressed as kilogrammes and metres, respectively. Body mass index (BMI) was calculated as follows: $\mathrm{BMI}=$ weight $/$ height $^{2}$. Physical activity was evaluated using the International Physical Activity Questionnaire (Hagströmer et al. 2006). Hand grip strength was determined using a Handheld Dynamometer (North Coast Medical, Morgan Hill) at both visits as described in detail elsewhere (Peolsson et al. 2001). Three trials with a rest of $30 \mathrm{~s}$ between the tests were allowed for each hand alternately and patients were encouraged to exert their maximal grip strength. The best result from these three tests was used for maximum handgrip strength. The intraclass correlation coefficients (ICC) for the left and the right hand were $98 \%$ for both hands in the young group and 98 and $96 \%$ in the old group, respectively.

Assessment of MVC force of musculus quadriceps femoris

The MVC force of the musculus quadriceps femoris was measured as described in detail by Maughan et al. (1983a). Briefly, subjects were seated in an adjustable, straight-backed chair with their hips and their knees flexed at $90^{\circ}$. The pelvis and the tights were secured by a broad strap and the patient was asked to cross their arms in front of their chests. A load cell (DFS Ametek Inc., Chatillon, USA), mounted on the chair frame, was connected to an inflexible strap placed around the ankle of the patient. After the instruction of the procedure and two to three practical trials, the patient was asked to extend the leg as strong as possible. The measurement was done three times and the best result was used for further analysis. The rest period between each trial was $2 \mathrm{~min}$. A force-time curve was obtained and analysed by NexygenTM DF Series Software (Ametek Inc., Chatillon, USA). Patients were asked to avoid sports exercise $48 \mathrm{~h}$ before the measurements. These strength measurements were performed at each visit of the patients. The ICCs for MVC force measurement were 98 and $95 \%$ for the young and the old group, respectively.

Assessment of morphological parameters with M-US

Patients lay supine with knees resting comfortably in extension near the natural resting position of $10-20^{\circ}$. A towel roll under the knee was used for positioning and patients were instructed to relax their muscles during the examination. All images were taken at $50 \%$ of the tight length from the greater trochanter to the lateral knee joint space as it has been shown that the anatomical CSA of the human quadriceps is at a maximum at about $50 \%$ of the femur length (Narici et al. 1989). Each midpoint was clearly marked on the skin with a surgical pen to ensure proper placement of the probe across repeated scans. A gel pad was used for all measures to avoid compression or deformation of the muscle. Two-dimensional B-mode ultrasound imaging with $7.25 \mathrm{MHz}$ linear array transducer (Siemens Acuson Antares) was used and images of musculus rectus femoris, musculus intermedius, 
musculus vastus lateralis and musculus vastus medialis were taken separately with the probe oriented in the transverse and the longitudinal plane perpendicular to the skin. The collected images were downloaded for further analysis with ImageJ (National Institutes of Health, USA). Maximal muscle thickness was measured in transversal images as the distance between the superficial and the deep fascia at the widest distance (Fig. 1a, b). The pennation angle was defined as the angle between muscle fibres and the deep fascia of the muscle. Pennation angles were therefore measured in the longitudinal ultrasound image for three fibres and the average of these three measurements was used for further analysis (Fig. 2a, b). As the orientation of the fibres of the rectus femoris was almost parallel to their fascia, no pennation angle was determined for this muscle of the musculus quadriceps. Echogenicity was determined using a computer assisted grey-scale analysis offered by ImageJ. In the longitudinal ultrasound image a region of interest was selected in each muscle without any bone or surrounding fascia. The mean echo intensity of this region was calculated $($ black $=0$, white $=255$ ).

\section{Statistical analysis}

All analyses were done using the SAS 9.2. System. The M-US parameters muscle thicknesses and echogenicity of musculus rectus femoris, musculus intermedius,

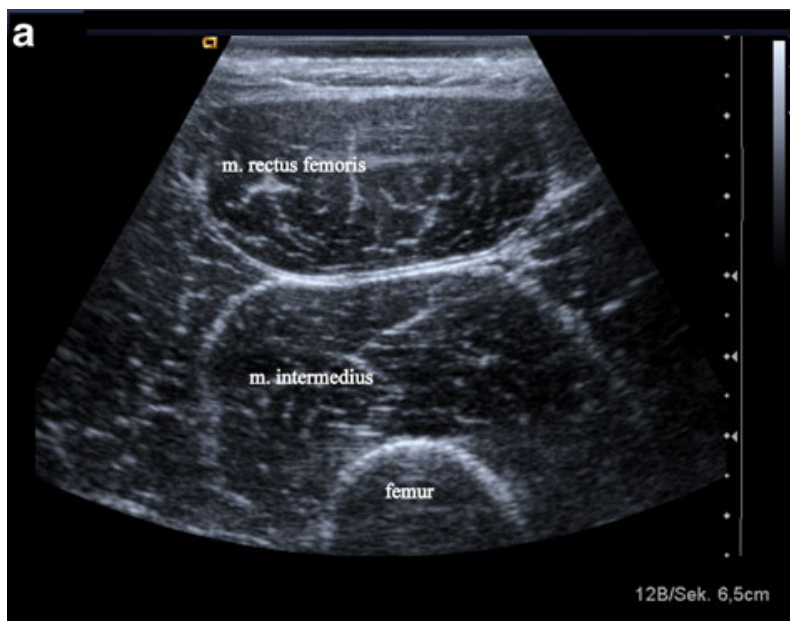

Fig. 1 Transversal ultrasound images of musculus rectus femoris and musculus intermedius from a 28 -year-old man (a) and a 69-year-old man (b). Maximal muscle thickness was musculus vastus lateralis and musculus vastus medialis as well as pennation angles of musculus intermedius, musculus vastus lateralis and musculus vastus medialis were investigated on two separate days. On each examination day, each observer measured these variables two times for each subject (four measurements per patient per day, altogether eight measurements per patient). MVC force of the musculus quadriceps as well as handgrip strength for the left and right hand were measured only once per day (altogether two measurements per patient).

For comparison of M-US measurements between young and old patients, mean values over all eight measurements per patient were calculated. The resulting averaged variables were described by mean \pm standard deviation and $t$ test was performed to investigate differences between the two groups.

For determination of reproducibility of M-US measurements, analysis of variance components with random-effect mixed models were used separately for the old and young group to determine ICCs, inter-day (within-subject, between-examination days), inter-rater (within-subject, between-observer) and inter-repetition (within subject, between repetition) standard deviations. The ICCs were calculated as the ratio of the inter-subject component of variance to the total variance. The inter-day, inter-rater and interrepetition standard deviations were calculated as square root of the corresponding variance components

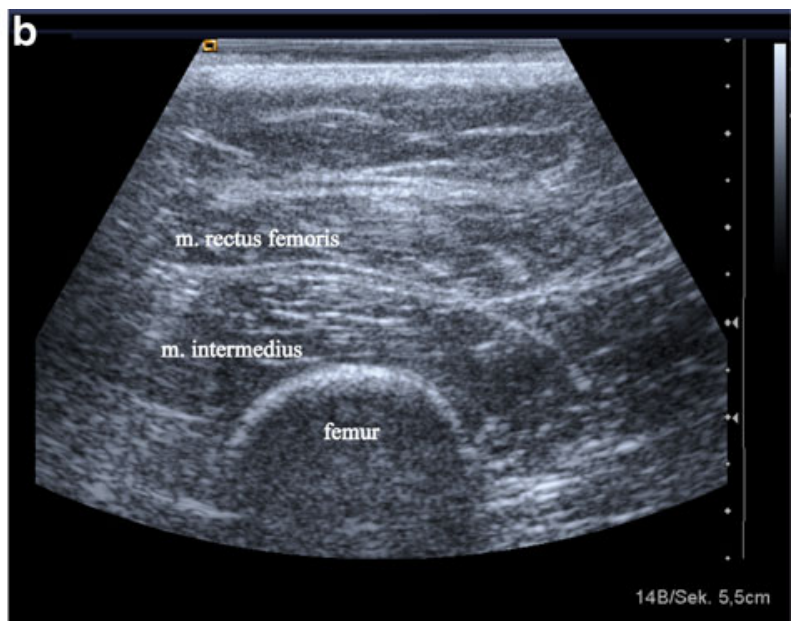

measured separately from musculus rectus femoris and musculus intermedius at the widest distance between the upper and the deep fascias 


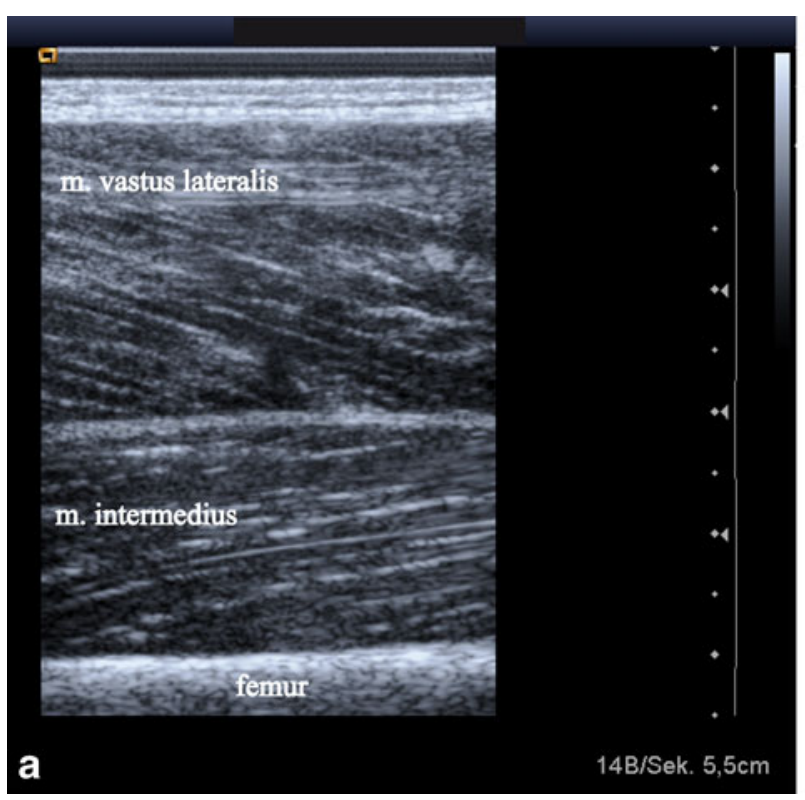

Fig. 2 Longitudinal ultrasound images of musculus vastus lateralis and musculus intermedius of a 23 -year-old man (a) and a 75 year-old-man (b). Pennation angles were measured

determined from the analysis. Furthermore, random effects mixed models (with random factor patient) were used to evaluate the effect of examination days, observer and repetition as well as age, sex, BMI and side (left/right leg) on M-US parameters. A $p$ value, corrected for multiplicity for the 11 investigated variables by Bonferroni correction, smaller than 0.0045 was considered as statistically significant.

To analyse the relationship between the averaged M-US parameters (mean over eight measurements per patient) and the averaged MVC force of musculus quadriceps (mean over two measurements per patient), first Pearson correlation coefficients were calculated separately for the young and the old groups.

To further investigate this relationship, simple random effects mixed models (with random factor patient) with target variable MVC of musculus quadriceps were calculated accounting for each of the $11 \mathrm{M}$-US parameters (using the per-day average over four measurements per patient) as well as for day, age, sex, BMI and side. Here, averages per day for each of the 11 variables were calculated since MVC was only measured once per day. Additionally, a multiple random effects mixed model (with random factor patient) was performed accounting for all influence variables with a univariate $p$ value smaller than 0.05 .

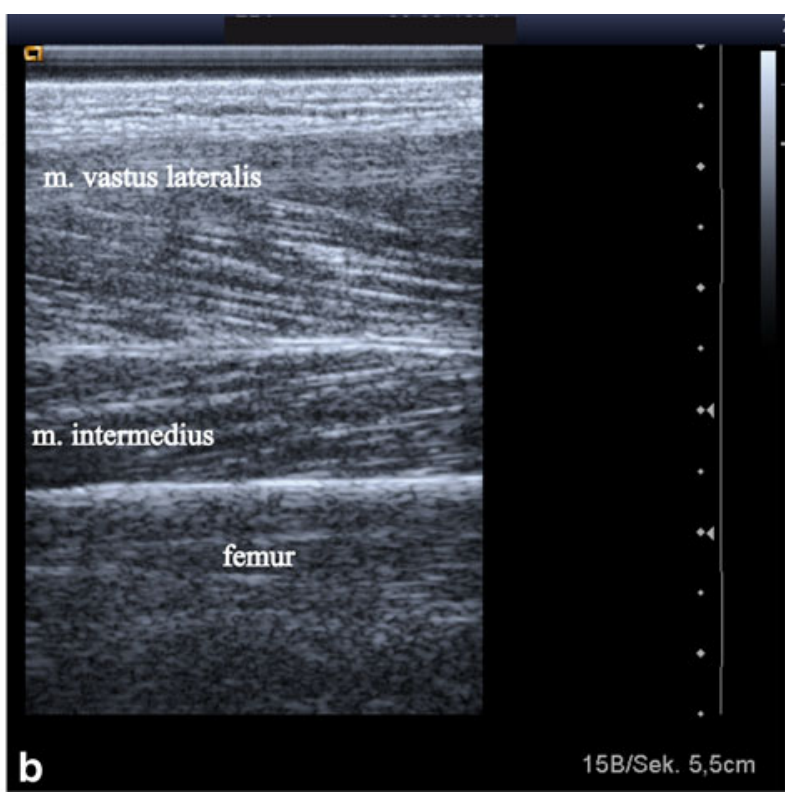

between muscle fibres and the deep fascia of the muscle. The same images were used for echogenicity measurements by computerassisted grey scale analysis

All statistical analyses were done separately for the old and the young group.

\section{Results}

A total of 52 patients were included in the study. The reasons for the patients to show up in the outpatient clinic were pain in the neck, the shoulder or the upper extremities. All patients were initially treated until the primary health problem was solved and afterwards enrolled in the study. Two patients in the young group and one patient of the old study group did not show up for the second measurement.

The young group had a mean age of $24.2 \pm 3.7$ years whereas the old group had a mean age of $67.8 \pm 4.8$ years. The mean height and weight was $1.8 \pm 0.1 \mathrm{~m}$ and $70.2 \pm$ $15.1 \mathrm{~kg}$ in the young group and $1.7 \pm 0.1 \mathrm{~m}$ and $77.2 \pm$ $13.2 \mathrm{~kg}$ in the old group. The mean BMI of the young and the old group were $22.5 \pm 3.0$ and $26.6 \pm 3.3 \mathrm{~kg} / \mathrm{m}^{2}$, respectively. The mean total physical activity score assessed by the International Physical Activity Questionnaire was 5,527.7 $\pm 5,386.6 \mathrm{MET}$-min/week in the young group and 7,192.7 $\pm 7,467.2 \mathrm{MET}$-min/week in the old group, revealing no significant difference between the groups. 
Measurement of MVC force of musculus quadriceps and handgrip strength

Measurement of MVC of the musculus quadriceps revealed a mean strength of $510.8 \pm 178.4 \mathrm{~N}$ in the young group. Overall, with a mean MVC of $352.1 \pm$ $114.2 \mathrm{~N}$, the old group was significantly weaker than the young group $(-31.1 \%, p<0.001)$.

The old group showed a significantly lower mean hand grip strength of the left $(32.0 \pm 11.4$ vs. $40.8 \pm$ $13.6 \mathrm{~kg}, p<0.05)$ and the right hand $(34.6 \pm 11.5 \mathrm{vs}$. $43.6 \pm 14.5 \mathrm{~kg}, p<0.05)$ compared to the young group.

Reproducibility of muscle thickness, pennation angle and echogenicity measurements with M-US

To investigate the reproducibility of the M-US measurements of muscle thickness, pennation angle and echogenicity, ICCs were calculated separately for the young and the old group (Table 1). Statistical analysis revealed that measurements of muscle thickness were highly reproducible in all muscles of musculus quadriceps in the young and the old group with ICCs between 85 and $97 \%$. Reproducibility of measurements of pennation angles revealed moderate results for musculus intermedius with ICCs of 78 and $74 \%$ in the young and old group, respectively. However, worse results were found for pennation angle measurements of musculus vastus lateralis and medialis in both groups. Reproducibility of echogenicity measurements revealed remarkable differences between young and old patients. Whereas ICCs of the young group ranged between 57 and $65 \%$, ICCs of the old group ranged between 20 and $31 \%$. Overall no systematic difference between examination days, observer or repetition was found for measurements of muscle thickness and pennation angle. Otherwise a systematic difference between examination days was found for echogenicity measurements of musculus intermedius and vastus medialis of the old group and a systematic difference between observers for echogenicity measurements of musculus intermedius was found in the young group.

Additionally, a multiple regression analysis was performed to investigate possible influences of age, sex, BMI and side (left/right leg) on muscle thickness, pennation angle and echogenicity. No significant influence of the examined side on any parameter was found in both groups. Sex showed a significant influence on thickness measurements,
Table 1 Reproducibility of M-US parameters of the young and old group. Analysis of variance components with random effect mixed models were calculated to determine inter-day, inter-rater, inter-repetition and inter-subject standard deviations. ICCs were calculated as the ratio of the inter-subject component of variance to the total variance

\begin{tabular}{|c|c|c|c|c|c|c|c|c|c|c|}
\hline & \multicolumn{2}{|c|}{ Inter-day SD } & \multicolumn{2}{|c|}{ Inter-rater SD } & \multicolumn{2}{|c|}{ Inter-repetition SD } & \multicolumn{2}{|c|}{ Inter-subject SD } & \multicolumn{2}{|l|}{$\mathrm{ICCs}$} \\
\hline & Young & Old & Young & Old & Young & Old & Young & Old & Young & Old \\
\hline \multicolumn{11}{|l|}{ Muscle thickness } \\
\hline Musculus rectus femoris & 0.14 & 0.19 & 0.15 & $<0.01$ & $<0.01$ & 0.15 & 3.98 & 1.88 & 97.2 & 87.6 \\
\hline Musculus intermedius & 0.21 & 0.13 & 0.21 & 0.25 & 0.10 & 0.20 & 4.35 & 2.91 & 97.8 & 92.8 \\
\hline Musculus vast. lateralis & 0.25 & 0.36 & 0.20 & 0.45 & $<0.01$ & 0.09 & 3.74 & 2.39 & 95.7 & 85.2 \\
\hline Musculus vast. medialis & $<0.01$ & $<0.01$ & 0.11 & 0.26 & 0.20 & 0.26 & 6.06 & 4.70 & 97.9 & 94.9 \\
\hline \multicolumn{11}{|l|}{ Pennation angle } \\
\hline Musculus intermedius & 0.23 & $<0.01$ & 0.22 & 0.42 & $<0.01$ & $<0.01$ & 2.53 & 2.15 & 78.3 & 74.1 \\
\hline Musculus vast. lateralis & 0.55 & 0.34 & 0.54 & $<0.01$ & 0.63 & 0.19 & 2.31 & 2.45 & 53.0 & 68.7 \\
\hline Musculus vast. medialis & 0.82 & 0.66 & 0.46 & 0.61 & $<0.01$ & 0.45 & 1.70 & 0.88 & 43.6 & 18.9 \\
\hline \multicolumn{11}{|l|}{ Echogenicity } \\
\hline Musculus rectus femoris & 7.43 & 14.75 & 0.72 & 0.39 & $<0.01$ & 1.69 & 12.09 & 10.91 & 57.1 & 30.7 \\
\hline Musculus intermedius & 8.36 & 14.53 & 4.40 & 4.27 & $<0.01$ & 3.28 & 18.50 & 12.13 & 65.0 & 31.3 \\
\hline Musculus vast. lateralis & 6.21 & 13.10 & 3.85 & 3.88 & 2.22 & $<0.01$ & 13.36 & 10.74 & 62.3 & 29.7 \\
\hline Musculus vast. medialis & 7.37 & 12.30 & 1.45 & 3.97 & 3.61 & 1.61 & 14.99 & 8.38 & 62.4 & 20.9 \\
\hline
\end{tabular}


revealing that all parts of the quadriceps were significantly thicker $(p<0.0001)$ in men than in women. Age showed a significant influence on thickness of musculus vastus lateralis in the old group, meaning that thickness of musculus vastus lateralis significantly decreased with increasing age $(p=0.0005)$. In the young group thickness of musculus vastus medialis increased significantly with increasing BMI $(p=0.0032)$.

Measurement of morphological parameters

of the musculus quadriceps femoris

Table 2 shows the results of the comparisons between the young and the old group in the per-patient averaged values of muscle thickness, pennation angle and echogenicity. In the old group mean muscle thickness was significantly thinner in all muscles of musculus quadriceps than in the young group. Overall, in comparison to the young group, thickness of musculus rectus femoris showed the greatest reduction for $25.4 \%$ in the old group whereas musculus intermedius, musculus vastus lateralis and vastus medialis in the old group were thinner for 14, 12.4 and $12 \%$, respectively. Moreover, pennation angles of musculus vastus lateralis $(-20.1 \%)$ and musculus vastus medialis $(-13.3 \%)$ were significantly lower in the old group than in the young group. No significant difference was found for pennation angles of musculus intermedius between the young and the old group. Comparing the results for echogenicity, a significantly higher echogenicity was determined in musculus rectus femoris $(+19.7 \%)$, musculus intermedius $(+31.8 \%)$, musculus vastus lateralis $(+15.7 \%)$ and vastus medialis $(+13.7 \%)$ of the old group compared to the young group.
The relationship between MVC force and M-US parameters

To investigate the correlation between MVC and muscle thickness, pennation angle and echogenicity, Pearson correlation coefficients were calculated (Table 3). Correlation coefficients between MVC and muscle thicknesses of the distinct muscles of musculus quadriceps were generally strong and significant, indicating that a higher MVC was associated with a higher muscle thickness in the young and in the old patient group (Fig. 3). Regarding the correlation between pennation angle and MVC, Pearson correlation coefficients demonstrated no significant correlation in the old group. In the young group, only pennation angle of the musculus intermedius was significantly and moderately strong correlated to MVC. Correlation coefficients for the relationship between echogenicity and MVC showed no significant correlations in the old group. In the young group moderate, significant negative correlations were found for all muscles of musculus quadriceps demonstrating that higher MVC was correlated with a lower echogenicity.

To further investigate which M-US parameter had a significant influence on MVC, simple random effect mixed models were calculated separately for the young and the old group. In both groups significant influences of muscle thicknesses of musculus rectus femoris $(p<0.00001)$, musculus intermedius $(p<0.00001)$, musculus vastus lateralis $(p<0.00001)$ and musculus vastus medialis $(p<0.00001)$ on MVC were found. Isometric maximum voluntary contraction force increased with increasing muscle thicknesses of all muscles of musculus quadriceps. In addition, MVC increased significantly with increasing values of the pennation angle

group and in the old group. Values are expressed as mean $\pm \mathrm{SD}$ over all measurements. For comparison between the young and the old group, $t$ test was performed for each parameter
Table 2 Muscle ultrasound parameters of the young and the old group. Muscle thickness, pennation angles and echogenicity of all parts of the musculus quadriceps were measured in the young

\begin{tabular}{|c|c|c|c|c|c|c|c|c|c|}
\hline & \multicolumn{3}{|c|}{ Muscle thickness (mm) } & \multicolumn{3}{|c|}{ Pennation angle $\left(^{\circ}\right)$} & \multicolumn{3}{|l|}{ Echogenicity } \\
\hline & Young & Old & $p$ & Young & Old & $p$ & Young & Old & $p$ \\
\hline Musculus rectus femoris & $18.1 \pm 4.0$ & $13.5 \pm 1.9$ & $<0.0001$ & - & - & & $101.9 \pm 14.0$ & $120.9 \pm 15.4$ & $<0.0001$ \\
\hline Musculus intermedius & $17.1 \pm 4.4$ & $14.7 \pm 2.9$ & $<0.05$ & $9.7 \pm 2.6$ & $8.8 \pm 2.2$ & 0.26 & $77.7 \pm 20.1$ & $102.4 \pm 16.5$ & $<0.0001$ \\
\hline Musculus vastus lateralis & $22.6 \pm 3.8$ & $19.8 \pm 2.4$ & $<0.01$ & $14.7 \pm 2.5$ & $11.9 \pm 2.5$ & $<0.0001$ & $96.2 \pm 14.9$ & $111.3 \pm 15.0$ & $<0.001$ \\
\hline Musculus vastus medialis & $29.9 \pm 6.1$ & $26.3 \pm 4.7$ & $<0.05$ & $12.1 \pm 2.0$ & $10.4 \pm 1.3$ & $<0.001$ & $94.3 \pm 17.8$ & $107.2 \pm 13$ & $<0.01$ \\
\hline
\end{tabular}


Table 3 Correlation coefficients between M-US parameters and MVC. Pearson correlation coefficients between muscle thickness, echogenicity, pennation angle and MVC were analysed. Results of the distinct muscles are presented for the young and the old group separately

\begin{tabular}{|c|c|c|c|c|c|c|c|c|}
\hline & \multicolumn{2}{|c|}{ Musculus rectus femoris } & \multicolumn{2}{|c|}{ Musculus intermedius } & \multicolumn{2}{|c|}{ Musculus vastus lateralis } & \multicolumn{2}{|c|}{ Musculus vastus medialis } \\
\hline & Young & Old & Young & Old & Young & Old & Young & Old \\
\hline Muscle thickness & $0.88^{*}$ & $0.834^{*}$ & $0.918 *$ & $0.815^{*}$ & $0.838^{*}$ & $0.756^{*}$ & $0.92 *$ & $0.875^{*}$ \\
\hline Pennation angle & $-0.63^{*}$ & -0.301 & $-0.64 *$ & -0.339 & $-0.50 *$ & -0.272 & $-0.47 * *$ & -0.10 \\
\hline Echogenicity & - & - & $0.68^{*}$ & 0.306 & 0.341 & 0.322 & 0.338 & 0.253 \\
\hline
\end{tabular}

$* p<0.001, * * p<0.05$

of musculus intermedius $(p<0.00003)$ in the young group. No significant influence of echogenicity on MVC was found in both groups. Other influencing factors were sex $(p<0.00001)$ in both groups, meaning men exhibited a significant higher MVC than women, and BMI $(p<0.00001)$ in the young group, meaning MVC increased with increasing BMI. Examined side
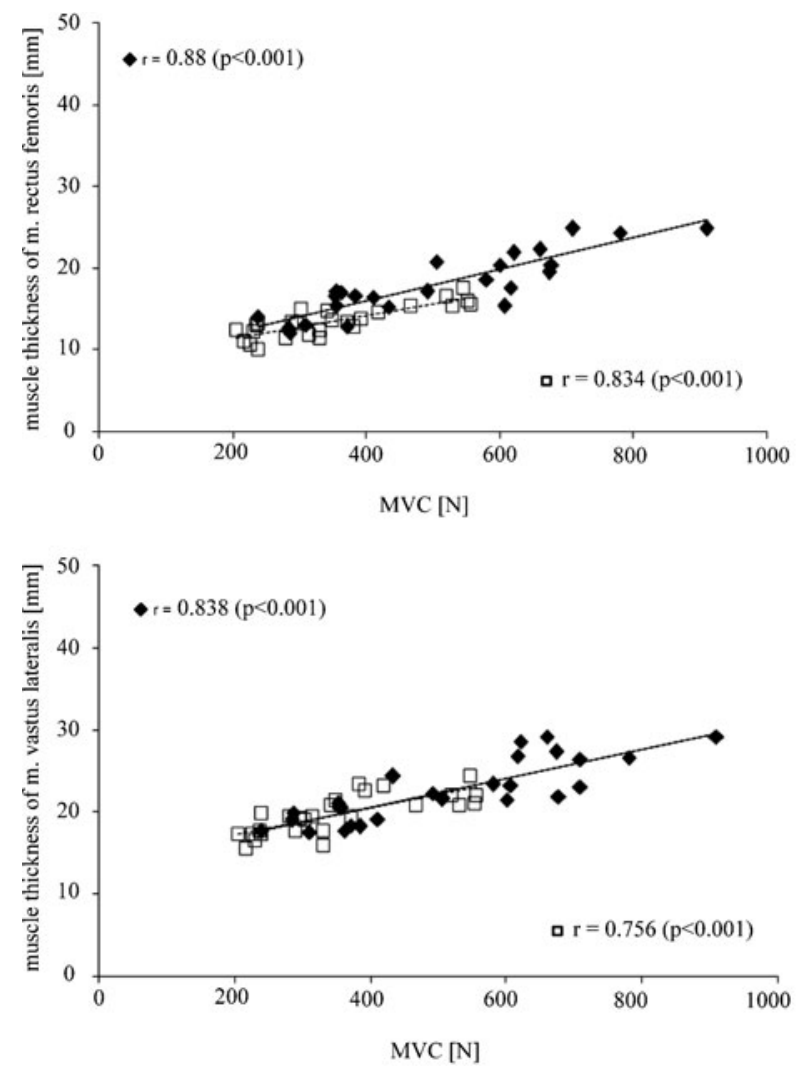

Fig. 3 Graphic presentation of the relationship between MVC and muscle thickness. Muscle thicknesses of musculus rectus femoris, musculus intermedius, musculus vastus lateralis and musculus vastus medialis were plotted against respective MVC of musculus quadriceps. The old patients are presented by open (left or right leg) and examination day showed no significant influence on MVC.

After exclusion of non-significant parameters, multiple regression models were performed for both groups. In the old group, only muscle thickness of musculus vastus medialis remained significant influence on MVC ( $p=0.027)$ whereas in the young group muscle
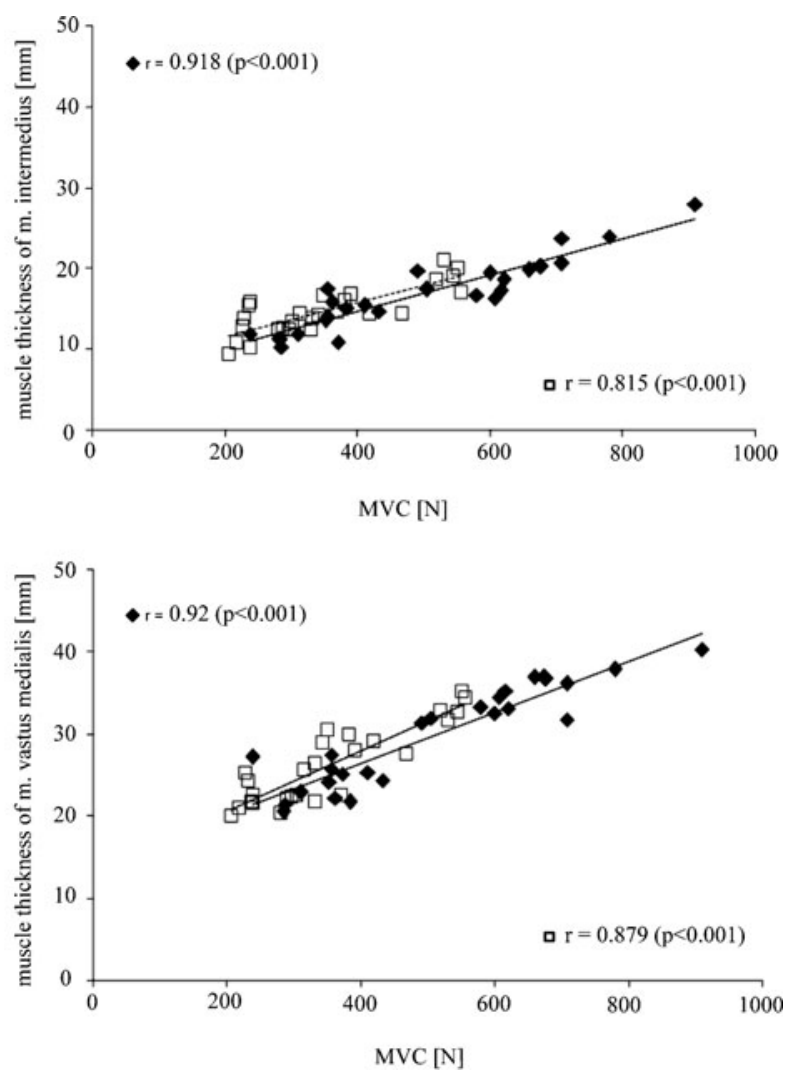

squares whereas the young patients are presented by closed squares. Pearson correlation coefficients as well as regression lines for the old (dotted line) and the young (straight line) patient group are shown 
thicknesses of musculus intermedius $(p=0.0385)$ and musculus vastus medialis $(p=0.0196)$ remained significant influence on MVC.

\section{Discussion}

The results presented herein revealed that M-US is a highly reproducible method to quantify muscle thicknesses of all muscles of musculus quadriceps both in young people and in old sarcopenic individuals. In addition, we found a strong correlation between the thickness of the muscles of musculus quadriceps and MVC. Moreover, this is the first study investigating reproducibility of additional M-US parameters as pennation angle and echogenicity of all muscles of musculus quadriceps in both young as well as sarcopenic muscles.

In the current study, muscle thickness measurements revealed the highest reproducibility with ICCs between 85 and $97 \%$. These results stand in accordance with other studies showing ICCs for muscle thickness measurements between 88 and $97 \%$ (Blazevich et al. 2006; Miyatani et al. 2002; Thoirs and English 2009) in various muscles.

Concerning the reproducibility of the pennation angle measurements we only found moderate ICCs of 74-78\% for measurements of musculus intermedius pennation angle. Reproducibility of pennation angle measurements of the other muscles of the musculus quadriceps was generally worse. Reasons for this finding might be difficulties in observer dependent techniques as pennation angles are strongly influenced by adjustment of the ultrasound probe. Our data are of high clinical impact because to our knowledge there is no comparable study in the literature investigating reproducibility of pennation angle measurements of all muscles of musculus quadriceps.

Analysis of reproducibility of muscle echogenicity measurements by M-US revealed moderate ICCs for the young group, although a systematic difference between observers for results of musculus intermedius were found. Moreover ICCs of echogenicity measurements in the old patient group were low and systematic differences between examination days were found for musculus intermedius and vastus medialis. Although we emphasised to attain the same examination and ultrasound equipment setting on both examination days, echogenicity measurements seems to be highly influenced by observer dependent factors as the adjustment of the ultrasound probe. Additionally, factors as for example the hydration balance of the muscle might also have an impact on echogenicity measurements. Regarding the literature data for reproducibility of echogenicity measurements in muscles, we found only one study investigating reproducibility of echogenicity of musculus musculus rectus femoris and intermedius in 145 healthy individuals between 20 and 90 years showing no interobserver reproducibility for measurements of echogenicity (Maurits et al. 2003). Therefore, further studies investigating reproducibility of echogenicity measurements and possible influencing factors are needed to optimise measurement techniques of this interesting parameter.

Skeletal muscle strength is an important predictor for patients's ability to return to activities of daily living after acute illness (Herridge et al. 2003) and for survival (Rantanen et al. 2000). Furthermore, skeletal muscle strength is an important parameter for diagnosis of sarcopenia. In this study, we intended to compare young subjects with sarcopenic subjects. Therefore, handgrip strength and MVC of musculus quadriceps of both groups were assessed. Handgrip strength of the left and the right hand was significantly reduced for 22 and $21 \%$, respectively, in the old group. Similarly, MVC of musculus quadriceps in the old group was significantly degraded for $31 \%$. These findings stand in accordance with the literature prescribing an annual loss of approximately $1.5 \%$ per year after the age of 50 (Doherty 2003; Ivey et al. 2000; Lauretani et al. 2003). Likewise in our study, Janssen et al. show that the lower extremities are more affected by muscle wasting than the upper extremities (Janssen et al. 2000). Although muscle strength and muscle mass changes during ageing are well described in the literature, there are only few publications investigating morphological changes as well as changes in muscle architecture and composition during ageing that can be determined with M-US. Therefore, our results add information about these changes in all muscles of musculus quadriceps during ageing. We found that thicknesses of all muscles of musculus quadriceps were significantly reduced in the old group compared to the young group. In the literature comparable results are found, showing a significantly thinner musculus vastus lateralis in elderly runners than in young runners (Karamanidis and Arampatzis 2006) 
and a reduction of muscle thickness of musculus rectus femoris and intermedius due to ageing (Maurits et al. 2003). Reimers et al. found a similar loss of muscle thickness of the musculus vastus lateralis of 15-21\% in 70-year-old subjects compared to 20-year-old subjects (Reimers et al. 1998). Similarly, our results showed that pennation angle of musculus vastus lateralis and medialis were significantly lower in the old group than in the young group. Although no publication about age dependent changes in pennation angles of musculus quadriceps were found in the literature, Morse et al. show a $12 \%$ decrease in pennation angle of musculus gastrocnemius in elderly patients (Morse et al. 2005). Concerning echogenicity measurements we found a significant increase in the old group, implicating that sarcopenic muscles contain more intramuscular fat and fibrous tissue than young muscles. This finding stands in accordance with the publication of Arts et al. describing that echogenicity of musculus rectus femoris increases with increasing age (Arts et al. 2010).

Concerning correlations between M-US parameters and MVC we found significant and moderate correlations ( $r=-0.47$ to -0.64 ) for echogenicity of all muscles of musculus quadriceps in the young group but no significant correlations in the old group. Our results of the correlation between echogenicity and MVC in rectus femoris of the old group were slightly worse $(r=-0.30$, n.s. $)$ than recent publications of Fukumoto et al. $(r=-0.40, p<0.01)$ and Cadore et al. $(r=-0.51$, $p<0.01$; Fukumoto et al. 2012; Cadore et al. 2012a). One explanation for this slight discrepancy might be the different modes of analysis. As both authors use transversal ultrasound images, we used longitudinal images for echogenicity determination. Although both methods are well described (Korhonen et al. 2009; Sipilä and Suominen 1996; Pillen et al. 2006), there is no direct comparison of these two methods for the investigated muscles in the literature. Investigations of pennation angle revealed a significant correlation with muscle strength only in the musculus intermedius of the young group whereas all other correlations were without significance. The strongest, significant correlations were detected between muscle thickness measurements and MVC in all measured subjects. Seymour et al. describe a similarly strong correlation $(r=0.78)$ between CSA of musculus rectus femoris and MVC in chronic obstructive pulmonary disease patients (Seymour et al. 2009) and Freilich et al. reveal a moderate correlation $(r=0.56)$ between MVC and the combined muscle thickness of musculus vastus lateralis and intermedius (Freilich et al. 1995). Moreover, a recent publication of Cadore et al. investigated neuromuscular adaptation of musculus quadriceps due to exercise training in elderly and found a significant increase in muscle thickness of all muscles of musculus quadriceps accompanied by an increase in one repetition maximum, maximal surface electromyographic activity and neuromuscular economy (Cadore et al. 2012b). However, our study for the first time compared thickness of all muscles of musculus quadriceps and MVC. Moreover, simple regression analysis revealed that in both groups thickness of all muscles of musculus quadriceps had a significant positive influence on MVC. In addition, pennation angle of musculus intermedius also had a significant positive influence on MVC in the young group. Otherwise, all other pennation angles as well as echogenicity had no significant influence on MVC in both groups. After multiple regression analysis, only thickness of musculus vastus medialis in the old group and thickness of musculus vastus medialis and musculus intermedius in the young group remained significant influence on MVC.

In conclusion, this study showed that thickness measurements of all muscles of musculus quadriceps were highly reproducible in young and sarcopenic muscles. Thickness measurements of all muscles of musculus quadriceps showed significant and high correlations to MVC of musculus quadriceps. Therefore, M-US muscle thickness measurement, especially of musculus intermedius and musculus vastus medialis, could be an accurate and bedside tool for diagnosis and course of sarcopenia in neuromuscular unimpaired patients. Further studies are needed to investigate whether M-US is able to detect muscle strength increases due to progressive muscle strength exercise in the elderly.

\section{References}

Abe T, Kawakami Y, Suzuki Y, Gunji A, Fukunaga T (1997) Effects of 20 days bed rest on muscle morphology. J Gravit Physiol 4:S10-S14

Arts IM, Pillen S, Schelhaas HJ, Overeem S, Zwarts MJ (2010) Normal values for quantitative muscle ultrasonography in adults. Muscle Nerve 41:32-41 
Blazevich AJ, Gill ND, Zhou S (2006) Intra- and intermuscular variation in human quadriceps femoris architecture assessed in vivo. J Anat 209:289-310

Cadore EL, Izquierdo M, Conceição M, Radaelli R, Pinto RS, Baroni BM, Vaz MA, Alberton CL, Pinto SS, Cunha G, Bottaro M, Kruel LF (2012a) Echo intensity is associated with skeletal muscle power and cardiovascular performance in elderly men. Exp Gerontol 47:473-478. doi:10.1016/j.exger.2012.04.002

Cadore EL, Izquierdo M, Pinto SS, Alberton CL, Pinto RS, Baroni BM, Vaz MA, Lanferdini FJ, Radaelli R, González-Izal M, Bottaro M, Kruel LF (2012b) Neuromuscular adaptations to concurrent training in the elderly: effects of intrasession exercise sequence. Age (Dordr). doi:10.1007/s11357-0129405-y

Cruz-Jentoft AJ, Baeyens JP, Bauer JM, Boirie Y, Cederholm T, Landi F, Martin FC, Michel JP, Rolland Y, Schneider SM, Topinková E, Vandewoude M, Zamboni M; European Working Group on Sarcopenia in Older People (2010) Sarcopenia: European consensus on definition and diagnosis: Report of the European Working Group on Sarcopenia in Older People. Age Ageing 39:412-423

Cooper R, Kuh D, Hardy R, Mortality Review Group, FALCon and HALCyon Study Teams (2010) Objectively measured physical capability levels and mortality: systematic review and meta-analysis. BMJ 341:c4467. doi:10.1136/ bmj.c4467

Doherty TJ (2003) Aging and sarcopenia. J Appl Physiol 95:1717-1727

Freilich RJ, Kirsner RL, Byrne E (1995) Isometric strength and thickness relationships in human quadriceps muscle. Neuromuscul Disord 5:415-422

Frontera WR, Hughes VA, Fielding RA, Fiatarone MA, Evans WJ, Roubenoff R (2000) Aging of skeletal muscle: a 12-yr longitudinal study. J Appl Physiol 88:1321-1326

Fukumoto Y, Ikezoe T, Yamada Y, Tsukagoshi R, Nakamura M, Mori N, Kimura M, Ichihashi N (2012) Skeletal muscle quality assessed from echo intensity is associated with muscle strength of middle-aged and elderly persons. Eur J Appl Physiol 112:1519-1525. doi:10.1007/s00421-0112099-5

Fukunaga T, Miyatani M, Tachi M, Kouzaki M, Kawakami Y, Kanehisa H (2001) Muscle volume is a major determinant of joint torque in humans. Acta Physiol Scand 172:249255

Gruther W, Benesch T, Zorn C, Paternostro-Sluga T, Quittan M, Fialka-Moser V, Spiss C, Kainberger F, Crevenna R (2008) Muscle wasting in intensive care patients: ultrasound observation of the $\mathrm{m}$. quadriceps femoris muscle layer. $\mathrm{J}$ Rehabil Med 40:185-189. doi:10.2340/16501977-0139

Hagströmer M, Oja P, Sjöström M (2006) The International Physical Activity Questionnaire (IPAQ): a study of concurrent and construct validity. Public Health Nutr 9:755-762

Herridge MS, Cheung AM, Tansey CM, Matte-Martyn A, DiazGranados N, Al-Saidi F, Cooper AB, Guest CB, Mazer CD, Mehta S, Stewart TE, Barr A, Cook D, Slutsky AS, Canadian Critical Care Trials Group (2003) One-year outcomes in survivors of the acute respiratory distress syndrome. N Engl J Med 348:683-693
Hughes VA, Frontera WR, Wood M, Evans WJ, Dallal GE, Roubenoff R, Fiatarone Singh MA (2001) Longitudinal muscle strength changes in older adults: influence of muscle mass, physical activity, and health. J Gerontol A Biol Sci Med Sci 56:B209-B217

Ikai M, Fukunaga T (1968) Calculation of muscle strength per unit cross-sectional area of human muscle by means of ultrasonic measurement. Int Z Angew Physiol 26:2632

Ivey FM, Tracy BL, Lemmer JT, NessAiver M, Metter EJ, Fozard JL, Hurley BF (2000) Effects of strength training and detraining on muscle quality: age and gender comparisons. J Gerontol A Biol Sci Med Sci 55:B152B157

Janssen I, Heymsfield SB, Wang ZM, Ross R (2000) Skeletal muscle mass and distribution in 468 men and women aged 18-88 yr. J Appl Physiol 89:81-88

Karamanidis K, Arampatzis A (2006) Mechanical and morphological properties of human quadriceps femoris and triceps surae muscle-tendon unit in relation to aging and running. $\mathrm{J}$ Biomech 39:406-417

Kawakami Y, Abe T, Fukunaga T (1993) Muscle-fiber pennation angles are greater in hypertrophied than in normal muscles. J Appl Physiol 74:2740-2744

Korhonen MT, Mero AA, Alén M, Sipilä S, Häkkinen K, Liikavainio T, Viitasalo JT, Haverinen MT, Suominen H (2009) Biomechanical and skeletal muscle determinants of maximum running speed with aging. Med Sci Sports Exerc 41(4):844-856. doi:10.1249/MSS.0b013e3181998366

Lauretani F, Russo CR, Bandinelli S, Bartali B, Cavazzini C, Di Iorio A, Corsi AM, Rantanen T, Guralnik JM, Ferrucci L (2003) Age-associated changes in skeletal muscles and their effect on mobility: an operational diagnosis of sarcopenia. J Appl Physiol 95:1851-1860

Manini TM, Clark BC, Nalls MA, Goodpaster BH, PloutzSnyder LL, Harris TB (2007) Reduced physical activity increases intermuscular adipose tissue in healthy young adults. Am J Clin Nutr 85:377-384

Maughan RJ, Watson JS, Weir J (1983a) Strength and crosssectional area of human skeletal muscle. J Physiol 338:3749

Maughan RJ, Watson JS, Weir J (1983b) Relationships between muscle strength and muscle cross-sectional area in male sprinters and endurance runners. Eur J Appl Physiol Occup Physiol 50:309-318

Maurits NM, Bollen AE, Windhausen A, De Jager AE, Van Der Hoeven JH (2003) Muscle ultrasound analysis: normal values and differentiation between myopathies and neuropathies. Ultrasound Med Biol 29:215-225

Miyatani M, Kanehisa H, Kuno S, Nishijima T, Fukunaga T (2002) Validity of ultrasonograph muscle thickness measurements for estimating muscle volume of knee extensors in humans. Eur J Appl Physiol 86:203-208

Morse CI, Thom JM, Reeves ND, Birch KM, Narici MV (2005) In vivo physiological cross-sectional area and specific force are reduced in the gastrocnemius of elderly men. $\mathrm{J}$ Appl Physiol 99:1050-1055

Narici MV, Roi GS, Landoni L, Minetti AE, Cerretelli P (1989) Changes in force, cross-sectional area and neural activation during strength training and detraining of the human quadriceps. Eur J Appl Physiol Occup Physiol 59:310-319 
Peolsson A, Hedlund R, Oberg B (2001) Intra- and inter-tester reliability and reference values for hand strength. J Rehabil Med 33:36-41

Pillen S, van Keimpema M, Nievelstein RA, Verrips A, van Kruijsbergen-Raijmann W, Zwarts MJ (2006) Skeletal muscle ultrasonography: visual versus quantitative evaluation. Ultrasound Med Biol 32:1315-1321

Pillen S, Tak RO, Zwarts MJ, Lammens MM, Verrijp KN, Arts IM, van der Laak JA, Hoogerbrugge PM, van Engelen BG, Verrips A (2009) Skeletal muscle ultrasound: correlation between fibrous tissue and echo intensity. Ultrasound Med Biol 35:443-446. doi:10.1016/ j.ultrasmedbio.2008.09.016

Rantanen T, Guralnik JM, Foley D, Masaki K, Leveille S, Curb JD, White L (1999) Midlife hand grip strength as a predictor of old age disability. JAMA 281:558-560

Rantanen T, Harris T, Leveille SG, Visser M, Foley D, Masaki K, Guralnik JM (2000) Muscle strength and body mass index as long-term predictors of mortality in initially healthy men. J Gerontol A Biol Sci Med Sci 55:M168M173

Reimers CD, Harder T, Saxe H (1998) Age-related muscle atrophy does not affect all muscles and can partly be compensated by physical activity: an ultrasound study. J Neurol Sci 159:60-66
Reimers K, Reimers CD, Wagner S, Paetzke I, Pongratz DE (1993) Skeletal muscle sonography: a correlative study of echogenicity and morphology. J Ultrasound Med 12:73-77

Rutherford OM, Jones DA (1992) Measurement of fibre pennation using ultrasound in the human quadriceps in vivo. Eur J Appl Physiol Occup Physiol 65:433-437

Seymour JM, Ward K, Sidhu PS, Puthucheary Z, Steier J, Jolley CJ, Rafferty G, Polkey MI, Moxham J (2009) Ultrasound measurement of rectus femoris cross-sectional area and the relationship with quadriceps strength in COPD. Thorax 64:418-423

Sipilä S, Suominen H (1996) Quantitative ultrasonography of muscle: detection of adaptations to training in elderly women. Arch Phys Med Rehabil 77:1173-1178

Taekema DG, Gussekloo J, Maier AB, Westendorp RG, de Craen AJ (2010) Handgrip strength as a predictor of functional, psychological and social health. A prospective population-based study among the oldest old. Age Ageing 39:331-337

Thoirs K, English C (2009) Ultrasound measures of muscle thickness: intra-examiner reliability and influence of body position. Clin Physiol Funct Imaging 29:440-446

Young A, Stokes M, Crowe M (1985) The size and strength of the quadriceps muscles of old and young men. Clin Physiol $5: 145-154$ 